\title{
DISMINUCIÓN DEL CONTENIDO DE POTASIO Y FÓSFORO EN BANANO (Musa sapientum L) PARA PACIENTES CON ENFERMEDAD RENAL CRÓNICA
}

\section{DECREASE OF POTASSIUM AND PHOSPHORUS CONTENT IN BANANA (MuSa sapientum $L$ ) FOR PATIENTS WITH CHRONIC RENAL DISEASE}

\author{
Rueda P. Elsy V.1 ${ }^{\star}$, Maldonado O. Yohanna ${ }^{2}$, Luna G. Nidia Y. ${ }^{1}$ \\ ${ }^{1 *}$ Universidad de Pamplona, Facultad de Salud, Grupo de Investigación de Salud Humana, \\ e-mail:elsyrueda@unipamplona.edu.co;e-mail:nluna@unipamplona.edu.co \\ ${ }^{2}$ Universidad de Pamplona, Facultad de Educación, Grupo de Investigación GIBA, e-mail: johamalob@gmail.com
}

Recibido 21 de Julio 2016; aceptado 30 de octubre de 2016

\section{RESUMEN}

La insuficiencia renal crónica es un problema de salud pública a nivel mundial, que se viene incrementando cada día más. Las personas con este tipo de patología deben seguir una dieta baja en potasio. Para ello, las guías alimentarias para la enfermedad renal crónica recomiendan limitar el consumo de muchas frutas, así como recurrir a métodos culinarios que permitan disminuir al máximo la cantidad de potasio presente en los alimentos. Por tal motivo, el objetivo de este trabajo fue analizar la reducción de potasio del banano sometido a remojo en diferentes tiempos (0,12 y 24 horas). Se analizó el contenido de potasio de las muestras por duplicado mediante espectrometría de absorción atómica de llama. Los resultados mostraron reducciones significativas en el contenido de potasio $(12,36 \%$ entre las 0 y 12 horas de remojo, 35,01\% entre las 12 y 24 horas y un 56,95\% 
Autor a quien dirigirse la correspondencia * Correo electrónico: elsyrueda@unipamplona.edu.co después de 24 horas) y de fósforo $(0.11 \%, 32.07 \%$ y $32.15 \%$ respectivamente) en los diferentes tiempos de remojo. Los resultados mostraron que es posible disminuir el contenido de potasio y fósforo en diferentes tiempos de remojo y los datos obtenidos sirven como referente a los profesionales que tratan con este tipo de pacientes. Se recomienda seguir este tipo de investigaciones utilizando diferentes técnicas culinarias que proporciones alternativas de consumo de alimentos para pacientes con enfermedad renal crónica.

Palabras clave: Banano, Enfermedad Renal Crónica, Fósforo, Potasio, Proceso Culinario.

\section{ABSTRACT}

Chronic renal failure is a worldwide public health problem, which is increasing every day. People with this type of pathology should follow a diet low in potassium. To do this, dietary guidelines for chronic kidney disease recommend limiting the consumption of many fruits, as well as using culinary methods to minimize the amount of potassium present in food. For this reason, the objective of this work was to analyzer the reduction of potassium of the banana soaked in different times (0,12 and 24 hours). The potassium content of the samples was analyzed in duplicate by flame atomic absorption spectrometry. The results showed significant reductions in potassium content (12.36\% between 0 and 12 hours of soaking, $35.01 \%$ between 12 and 24 hours and $56.95 \%$ after 24 hours) and phosphorus $(0.11 \% \%, 32.07 \%$ and $32.15 \%$, respectively) in the different soaking times. The results showed that it is possible to decrease potassium and phosphorus content in different soaking times and the data obtained serve as a reference for professionals dealing with this type of patients. It is recommended to follow this type of research 
using different culinary techniques that provide alternative food consumption for chronic kidney patients.

Key words: Banana, Chronic Kidney Disease, Phosphorus, Potassium, Culinary Process.

\section{INTRODUCCIÓN}

La Enfermedad Renal Crónica (ERC) es un problema creciente a nivel mundial, con una prevalencia estimada que se aproxima al 10\% (Coresh et al., 2005). El deterioro de la función renal provoca la retención de sustancias como el fósforo y el potasio, que presentan un mecanismo de adaptación a la pérdida funcional de nefronas a través del balance glomérulo-tubular. La retención de fósforo ocurre desde estadios tempranos de la ERC (probablemente en el estadio 1 y en forma definitiva en el estadio 2) apareciendo en forma de hiperfosfaturia y posteriormente hiperfosfatemia en estadios 4-5, asimismo, ocurre con el potasio. (National Kidney Foundation, 2003; Torregrosa et al., 2011).

Dentro de los objetivos del tratamiento dietético en pacientes con Enfermedad Crónica Renal se pueden resaltar los siguientes: proporcionar suficientes proteínas para compensar las pérdidas de aminoácidos esenciales y nitrógeno, controlar el potasio sérico para prevenir la hipercaliemia y las arritmias cardíacas; limitar el fósforo para controlar la hiperfosfatemia y reducir al mínimo la osteodistrofia renal. (Klahr S., 1991). Debido a ello, los pacientes con ERC tienen requerimientos nutricionales específicos. En el caso del potasio, su ingesta debe limitarse a 1.500-2.000 mg/día (López et al., 2008;

Cano et al., 2009), según el estadio de la enfermedad y la situación específica de cada paciente. Con el fin de prevenir una posible hiperpotasemia, los pacientes deben seguir una dieta baja en potasio, evitando alimentos cuyo contenido natural en potasio sea elevado, o aquellos que contengan aditivos con sales potásicas (Saxena A., 2012). Para tal fin se han generado diferentes métodos para preparación de alimentos que permitan que los pacientes con esta patología puedan consumirlos (KDIGO, 2009). Dentro de los alimentos que se recomiendan para su nutrición están las frutas y verduras frescas que se consumen comúnmente debido a su disponibilidad, fácil, procesamiento, sabor y particularmente debido a su efecto beneficioso sobre la salud humana. Estos productos son fuente natural de antioxidantes y otros compuestos bioactivos (Singh et al., 2015), 
especialmente constituyentes fenólicos, flavonoides, antocianidinas, vitamina C, minerales y carotenoides que influyen positivamente en la salud humana $e$ indican una alta actividad antioxidante (Viuda-Martos et al., 2010; ContrerasCalderón et al., 2011; Martínez et al., 2012). Algunas frutas como el banano ofrecen grandes beneficios médicos. (Someya et al., 2002; Davey et al., 2007; Fungo y Pillay, 2010) Esto es en parte debido a que es una rica fuente de importantes fitonutrientes, incluyendo vitaminas y compuestos fenólicos (Wall, 2006; Lim et al., 2007). También está notablemente enriquecida con minerales, tales como fósforo, sodio, potasio, calcio, magnesio, hierro, cobre, zinc y manganeso (Forster et al., 2003). Múltiples investigaciones han demostrado que sus componentes ayudan a la retención de calcio, nitrógeno y fósforo en el cuerpo, los cuales contribuyen en la reconstrucción de tejidos y puede ser utilizado para combatir los desórdenes intestinales como las úlceras, incluso las hojas de la platanera se pueden utilizar como una compresa fría para quemaduras y/o heridas (Kumar et al., 2012). Se observó que la pulpa de banano

\section{MATERIALES Y MÉTODOS}

\section{Material.}

Se adquirieron en el mercado local 10 unidades de banano común maduro, de los cuales se seleccionaron 5 bananos (Musa contiene compuestos bioactivos, como ácidos fenólicos y flavonoides con alto potencial antioxidante y actividad antitumoral (Borges et al., 2014), contribuye a reducir el riesgo de trastornos neurodegenerativos, retrasan el proceso de envejecimiento y ayudan a disminuir la incidencia de enfermedades degenerativas, como enfermedades del corazón, arteriosclerosis, inflamación, artritis, cáncer y disfunción cerebral (Singh et al., 2015). Debido a su alto contenido de potasio (328 mg) (ICBF, 2015), no es un alimento que sea de alto consumo para los ERC, por lo tanto, estudios recientes muestran que de acuerdo al método de cocción (vapor, asado, hervido, freído, salteado, utilización de Microondas y la cocción a presión) y preparación de los alimentos previamente (lavado, pelado, cortar, cortar y sembrar) se mejora la disponibilidad de los nutrientes sanos para el organismo. (Tiwari et al., 2013; Severi et al., 1998). El objetivo de la presente investigación fue analizar la disminución del contenido de potasio y fósforo en el banano por un método culinario como alternativa de consumo para los pacientes con deficiencia renal crónica. sapientum L) agrupados de acuerdo al estado de maduración número 5 de acuerdo a lo establecido en la NTC 1190 (ICONTEC, 1976; Aurore, et al., 2009) y con un peso promedio de $100 \mathrm{~g}$. Se les 
retiró la cáscara y a continuación cada uno de los bananos fue cortado en rodajas con un cortador en acero inoxidable de $5 \mathrm{~mm}$ de grosor.

Métodos. Luego de tener los frutos de banano seleccionados, fueron sumergidos en $200 \mathrm{ml}$ de agua en tiempos diferentes: sin remojo (0 horas), a las 12 horas de remojo y a las 24 horas de remojo, retirando el agua. Posteriormente se analizó el contenido de potasio de las muestras sometidas a cada tratamiento por medio de espectroscopia de absorción atómica utilizando un espectrofotómetro de absorción atómica (EAA) SHIMADZU AA
7000 con llama aire-acetileno de cátodo hueco para determinar $\mathrm{P}$ y K. por el método de la AOAC (2005). Los contenidos en potasio se expresaron en $\mathrm{mg} / 100 \mathrm{~g}$ por porción comestible (PC).

\section{Análisis Estadístico.}

Las diferencias estadísticas en el contenido en potasio tras la aplicación de las diferentes técnicas culinarias para cada tipo de muestra se establecieron mediante un análisis de variancia de un factor (ANOVA) Los distintos análisis estadísticos se llevaron a cabo utilizando Microsoft Excel 2016.

\section{RESULTADOS Y DISCUSIÓN}

Se observó una pérdida de potasio tras la aplicación de los procesos culinarios en diferentes tiempos de remojo en el banano.

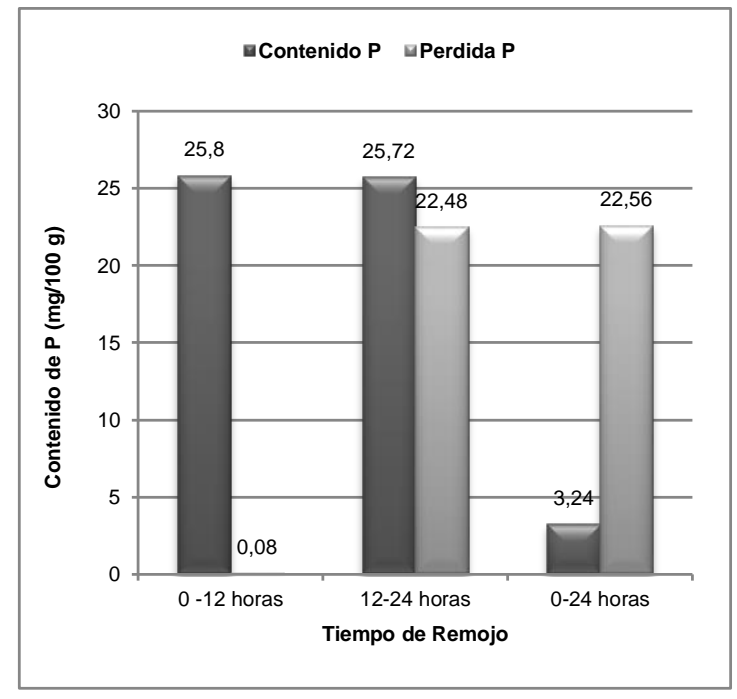

Figura 1. Pérdida de Potasio en los diferentes tiempos de remojo. Fuente: Autores.
En la figura 1 se muestra el contenido final de potasio (mg/100 $\mathrm{g}$ de muestra) y se observa que el porcentaje de pérdida contenido de potasio entre las 0 y 12 horas de remojo fue de $12,36 \%$, entre las 12 y 24 horas fue de $35,01 \%$ y un $56,95 \%$ de las 0 a 24 horas.

El banano analizado en la presente investigación presentó un contenido de potasio de $349,733 \mathrm{mg}$, el cual se acerca al relacionado por la tabla nutricional del Instituto Colombiano de Bienestar Familiar (ICBF, 2015) que establece que el banano contiene $358 \mathrm{mg}$ de potasio, observando en los resultados obtenidos que si la fruta se somete a 24 horas de remojo alcanza a perder más del $50 \%$ del contenido de 
potasio, posiblemente debido a la transferencia del mineral que posee la fruta al agua; por lo tanto se recomienda desechar el agua de remojo y no utilizarla para la preparación del alimento.

La figura 2 muestra que a partir de las 12 horas de remojo hubo una disminución del fósforo en el banano de un 32,07 \%, asimismo, presenta una reducción de un $32,15 \%$ después de 24 horas. Caso contrario a lo que se observa en el transcurso de las 0 a las 12 horas donde no hubo una disminución representativa del mineral $(0,11 \%)$.

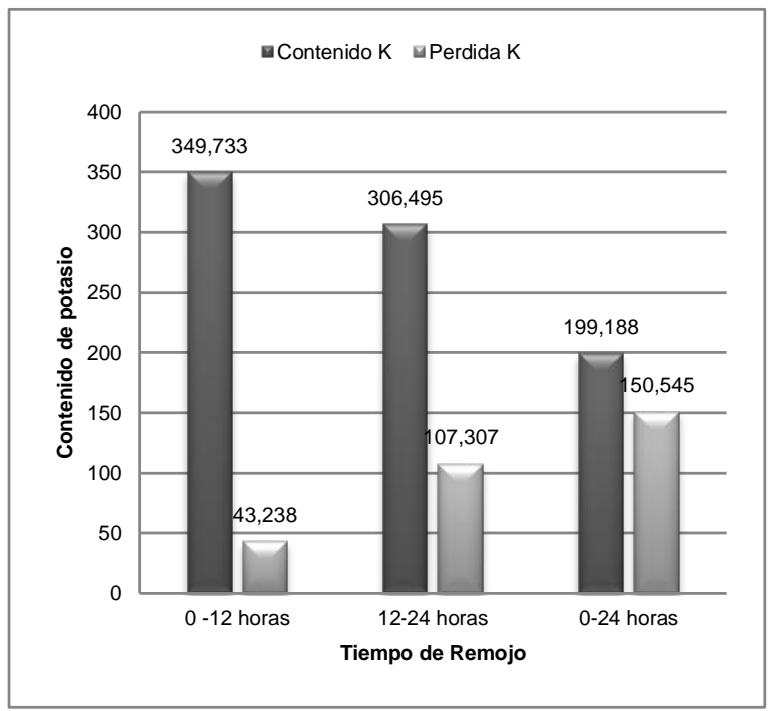

Figura 2. Pérdida de Fósforo en los diferentes tiempos de remojo.

Aurore et al., 2009 reporta que el banano contiene $22 \mathrm{mg}$ de fósforo y la Tabla Nutricional de alimentos del ICBF reporta un contenido de $27 \mathrm{mg}$, datos que no coinciden con los obtenidos en la presente investigación pero se acercan, donde se obtuvo un contenido de fósforo de $25,8 \mathrm{mg}$ en el banano analizado. Lo anterior se pude presentar por la variabilidad que existe en el cultivo de banano, características geográficas y nutricionales del suelo $\mathrm{y}$ deficiencia de nutrientes en la planta durante su cosecha. (Espinosa y Mite., 2002), características que no fueron tenidas en cuenta en la presente investigación.

Múltiples estudios han demostrado que la aplicación de diferentes métodos culinarios y previa preparación de los mismos como herramienta para la disminución de potasio $y$ fósforo en alimentos que sean de consumo en pacientes con enfermedad renal crónica son efectivos (Burrowes, 2008), sin embargo, muy pocos relacionados con frutas. Se han analizado vegetales como el brócoli (Martínez Hernández et al., 2013; Bongoni et al., 2014), la cebolla (Lee et al., 2008; Németh et al., 2003) papa (García-Segovia et al., 2008; Micklander et al., 2008) y leguminosas como frijoles (Saikia et al., 1999) y garbanzos (Duhan et al., 2002; Koplık et al., 2004) concluyendo que se producen pérdidas de nutrientes en las fases de preparación y cocción encontrando diferencias significativas entre los métodos de cocina utilizados y su influencia en el beneficio del organismo. (Kahlon et al., 2007). 


\section{CONCLUSION}

Los resultados obtenidos en el presente estudio confirman que el método de remojar previamente el banano para posteriormente procesarlo y prepararlo al gusto, reduce su contenido en potasio y fósforo hasta niveles aceptables, lo que permitiría su inclusión en la dieta de los pacientes con Enfermedad Renal Crónica, representando una alternativa de alimento para estos pacientes.

\section{AGRADECIMIENTOS.}

A la auxiliar Técnico del Laboratorio de Control de Calidad de la Universidad de
Pamplona Yolanda Rico y a las estudiantes de nutrición quienes prestaron su valiosa colaboración.

\section{REFERENCIAS BIBLIOGRÁFICAS}

Aurore, G., Parfait, B., \& Fahrasmane, L. (2009). Bananas, raw materials for making processed food products. Trends in Food Science \& Technology, 20, 78-91.

Bongoni, R., Verkerk, R., Steenbekkers, B., Dekker, M., Stieger, M., (2014). Evaluation of different cooking conditions on broccoli (Brassica oleracea var. italica) to improve the nutritional value and consumer acceptance. Plant Foods Hum. Nutr. 69 (3), 228-234.

Borges, C. V., de Oliveira Amorim, V. B., Ramlov, F., da Silva Ledo, C. A., Donato, M., Maraschin, M., \& Amorim, E. P. (2014). Characterisation of metabolic profile of banana genotypes, aiming at biofortified Musa spp. cultivars. Food chemistry, 145, 496-504.
Burrowes JD, Ramer NJ. (2008). Changes in potassium content of different potato varieties after cooking. $J$ Ren Nutr. 18:530-4).

Cano NJM, Aparicio M, Brunori G, Carrero JJ, Cianciaruso B, Fiaccadori E, et al., (2009) ESPEN guidelines on parenteral nutrition: Adult renal failure. Clin Nutr. 28:401-14.

Contreras-Calderón, J., Calderón-Jaimes, L., Guerra-Hernández, E., García-Villanova, B., 2011. Antioxidant capacity, phenolic content and vitamin $C$ in pulp, peel and seed from 24 exotic fruits from Colombia. Food Res. Int. 44 (7), 2047-2053.

Coresh J, Byrd-Holt D, Astor B, Briggs J, Eggers P, Lacher D et al., (2005). Chronic kidney disease awareness, prevalence 
and trends among U.S. adults, 1999 to 2000. J Am Soc Nephrol. 16: 180-188).

Davey, M. W., Van den Bergh, I., Markham, R., Swennen, R., \& Keulemans, J. (2009). Genetic variability in Musa fruit provitamin A carotenoids, lutein and mineral micronutrient contents. Food Chemistry, 115, 806-813.

Duhan, A., Khetarpaul, N., Bishnoi, S., 2002. Content of phytic acid and HClextractability of calcium, phosphorus and iron as affected by various domestic processing and cooking methods. Food Chem. 78, 9-14.

Espinosa, J., \& Mite, F. (2002). Estado actual y futuro de la nutrición y fertilización del banano. Revista Informaciones Agronómicas, 48, 4-9.)

Forster, M., Rodríguez, E. R., Martín, J. D., \& Romero, C. D. (2003). Distribution of nutrients in edible banana pulp. Food Technology and Biotechnology, 41, 167172.

Fungo, R., \& Pillay, M. (2013). $\beta$-Carotene content of selected banana genotypes from Uganda. African Journal of Biotechnology, 10, 5423-5430.

García-Segovia, P., Andrés-Bello, A., Martínez-Monzó, J., 2008. Textural properties of potatoes (Solanum tuberosum L., cv. Monalisa) as affected by different cooking processes. J. Food Eng. 88 (1), 28-35.

Instituto Colombiano de Bienestar Familiar ICBF. (2015) Tabla Nutricional de Alimentos. Colombia.

ICONTEC. Norma Técnica Colombiana NTC $1190 \quad$ (1976-12-17). Plátanos. Clasificación. E. Bananas. Classification.

Kahlon, T.S., Chiu, M.-C.M., Chapman, M.H., 2007. Steam cooking significantly improves in vitro bile acid binding of beets, eggplant, asparagus, carrots, green beans, and cauliflower. Nutr. Res. 27 (12), 750-755.

KDIGO (2009). Kidney Disease: Improving Global Outcomes (KDIGO) CKD-MBD Work Group.KDIGO clinical practice guideline for the diagnosis, evaluation, prevention, and treatment of chronic kidney disease - mineral and bone disorder (CKD-MBD). Kidney Int Suppl. (113):S1-130).

Klahr S. (1991). Enfermedad renal. En: Organización Panamericana de la Salud, Instituto Internacional de Ciencias de la Vida. Conocimientos actuales sobre nutrición. 6a edición. Washington, DC: OPS/ILSI; 436-44 (Publicación científica 532).

Koplık, R., Mestek, O., Kom' ınková, J., Borková, M., Suchánek, M., 2004.' Effect of cooking on phosphorus and trace 
a.LIMENTECH CIENCIA Y TECNOLOGÍA ALIMENTARIA ISSN 1692-7125. Volumen 14, No. 2, p. 23 - 32, año 2016 Facultad de Ingenierías y Arquitectura Universidad de Pamplona

elements species in peas. Food Chem. 85 (1), 31-39.

Kumar, K. P. S. et al., (2012). Traditonal and Medicinal Uses of Banana, 1(3), 51-63).

Lee, S.U., Lee, J.H., Choi, S.H., Lee, J.S., Ohnisi-Kameyama, M., Kozukue, N., Levin, C.E., Friedman, M., 2008. Flavonoid content in fresh, homeprocessed, and light-exposed onions and in dehydrated. J. Agric. Food Chem. 56, 8541-8548.

Lim, Y. Y., Lim, T. T., \& Tee, J. J. (2007). Antioxidant properties of several tropical fruits: A comparative study. Food chemistry, 103, 1003-1008.

López M Ruperto, Cuadrado G Barril, Sellares V Lorenzo. (2008). Guía de nutrición en enfermedad renal crónica avanzada (ERCA). Nefrologia. 79-86.

Martínez-Hernández, G.B., ArtésHernández, F., Gómez, P. a, Artés, F., (2013). Quality changes after vacuumbased and conventional industrial cooking of kailan-hybrid broccoli throughout retail cold storage. LWT-Food Sci. Technol. 50 (2), 707-714.

Martínez, R., Torres, P., Meneses, M.A., Figueroa, J.G., Pérez-Álvarez, J.A., ViudaMartos, M., (2012). Chemical, technological and in vitro antioxidant properties of mango, guava, pineapple and passion fruit dietary fiber concentrate. Food Chem. 135, 1520-1526.

Micklander, E., Thybo, A.K., van den Berg, F., 2008. Changes occurring in potatoes during cooking and reheating as affected by salting and cool or frozen storage - a LF-NMR study. LWT - Food Sci. Technol. 41 (9), 1710-1719.

National Kidney Foundation. (2003). K/DOQI clinical practice guidelines for bone metabolism and disease in chronic kidney disease. Am J Kidney Dis. 42(4 suppl 3):S1-202

Németh, K., Takàcsova, M., Piskua, M.K., 2003. Effect of cooking on yellow onion quercetin. Pol. J. Food Nutr. Sci. 12 (53), 170-174.

Saikia, P., Sarkar, C.R., Borua, I., 1999. Chemical composition, antinutritional factors and effect of cooking on nutritional quality of rice bean [Vigna umbellata (Thunb; Ohwi and Ohashi)]. Food Chem. 67 (4), 347-352.

Saxena A. Nutritional problems in adult patients with chronic kidney disease: Clinical queries. Nephrology. 2012; 1: 222-35.).

Severi S, Bedogoit G, ZoboJi G P, Manzierj A M, Polj M, Gatti G, Battistioi N. (1998). Effects of home-based food preparation practices on the micronutrient content of 
foods. European Journal of Cancer Prevention.7, 331-335.

Singh, J. P., Kaur, A., Shevkani, K., \& Singh, N. (2015). Influence of jambolan (Syzygium cumini) and xanthan gum incorporation on the physicochemical, antioxidant and sensory properties of gluten-free eggless rice muffins. International Journal of Food Science \& Technology, 50, 1190-1197.

Someya, S., Yoshiki, Y., \& Okubo, K. (2002). Antioxidant compounds from bananas (Musa Cavendish). Food Chemistry, 79, 351-354.

Tiwari, U., Cummins, E., (2013). Factors influencing levels of phytochemicals in selected fruit and vegetables during preand post-harvest food processing operations. Food Res. Int. 50 (2), 497506.

Torregrosa JV, Bover J, Cannata Andía J, Lorenzo V, de Francisco ALM, Martínez I, et al. (2011). Recomendaciones de la Sociedad Española de Nefrología para el manejo de las alteraciones del metabolismo óseo mineral en los pacientes con enfermedad renal crónica (S.E.N-MM). Nefrologia. 31 (Suppl 1):332).

Viuda-Martos, M., Fernández-López, J., Pérez-Álvarez, J.A., 2010. Pomegranate and its many functional components as related to human health: a review. Comp. Rev. Food Sci. Food Saf. 9, 635-654.

Wall, M. M. (2006). Ascorbic acid, vitamin A, and mineral composition of banana (Musa sp.) and papaya (Carica papaya) cultivars grown in Hawaii. Journal of Food Composition and Analysis, 19, 434-445. 